\title{
ÉTUDE DE LA RÉPARTITION DE LYMNAEA TRUNCATULA DANS LE NORD-OUEST ALGÉRIEN ET DE SA RÉCEPTIVITÉ A FASCIOLA HEPATICA
}

\author{
M. MASSOT et K. SENOUCI-HORR*
}

RÉSUMÉ. Une étude préliminaire sur les habitats de Lymnaea truncatula Müller dans le nordouest de l'Algérie a permis de dénombrer 33 gîtes positifs représentés par des oueds, des sources, des marécages et des canaux d'irrigation. Malgré un polymorphisme de la coquille et un polymorphisme enzymatique, les limnées de huit de ces stations se sont avérées expérimentalement réceptives à Fasciola hepatica $\mathrm{L}$.

\section{Distribution of Lymnaea truncatula in the North-West of Algeria and study of its receptivity to Fasciola hepatica}

SUMMARY. A preliminary study on the habitats of Lymnaea truncatula Müller in the Nort-West of Algeria has permitted to number 33 positive lodgings represented by "oueds", sources, marshes and irrigation canals. Despite a polymorphism of the shell and an enzymatic polymorphism, the snails of eight stations have proved experimentally receptive to Fasciola hepatica L.

\section{Introduction}

Les données sur la biologie de Fasciola hepatica et de son Mollusque vecteur en Algérie sont actuellement très peu nombreuses. Lymnaea truncatula a été signalé, en compagnie d'autres Lymnaeidés, à l'état fossile dans les couches sédimentaires du Sud des Hauts-Plateaux (Laghouat, El-Bayad, Djelfa) par Bourguignat (1862). Pallary $(1921,1926 \mathrm{a}, 1926 \mathrm{~b}, 1927)$ a rencensé 24 Basommatophores dont six Lymnaeidés (L. stagnalis, L. palustris, L. auricularia, R. limosa, L. glabra, L. truncatula) dans des biotopes du Tell et de l'Atlas. Dupouy et al. (1980) signale Lymnaea truncatula dans l'Oued Isser (Ouled Mimoun, Tlemcen). De ces rares données, on peut conclure que la distribution de Lymnaea truncatula en Algérie n'est pas connue, pas plus que dans le reste du Maghreb d'ailleurs. Les seules informations dont on dispose sur Fasciola hepatica sont des prévalences très anciennes portant sur les bovins et les ovins réunis (Lièvre, 1932) : Oranie (1\%), Algérois (3\%), Constantinois (12\%).

* Département de Biologie animale, Université d'Oran, Oran ès Senia, Algérie.

Accepté le 7 juillet 1982 . 
Des cas de fasciolose humaine ont été signalés dans ces régions (Senevet et Champagne, 1928-1929 ; Guy et al., 1969). Pour notre part, nous avons noté un foyer actif apparemment important de fasciolose chez les ovins dans la région de Saïda.

Le cycle de Fasciola hepatica dans le Maghreb n'a pas été étudié, qu'il s'agisse des modalités de développement, de la réceptivité des vecteurs, des sites de transmission ou des variations saisonnières ; l'écologie de la fasciolose n'est donc actuellement connue en détail que pour des régions ayant des caractères bioclimatiques très différentes de l'Afrique du Nord.

Dans ce travail, nous abordons le problème des gîtes à limnées et de la valeur de ces dernières en tant qu'hôtes intermédiaires.

\section{Distribution géographique}

Nous avons prospecté le Nord-Ouest algérien y compris une grande partie des Hauts-Plateaux. La zone de nos recherches s'inscrit entre Alger, Djelfa, Aflou, Bougtob, El-Aricha, la frontière algéro-marocaine et la côte méditerranéenne. Cette aire de prospection s'étend à l'intérieur du pays sur des distances de $130 \mathrm{~km}$ (au niveau de la frontière algéro-marocaine), à $280 \mathrm{~km}$ (à la latitude d'Alger).

Parmi 146 points contrôlés (101 oueds, 17 sources, 16 marécages et 12 canaux), nous avons répertorié 33 gîtes à Lymnaea truncatula. La distribution des gîtes ( ig. 1) met en évidence l'importance de certains bassins hydrographiques; c'est le cas de la Tafna qui prend sa source près de Seddou et draîne les régions de Tlemcen et de Maghnia, des alentours de Bel-Abbès qui regorgent d'oueds et de terrains marécageux, de la région de Saïda (altitude $800-1000 \mathrm{~m}$ ) qui offre de nombreux points d'eau; cette dernière région est un lieu d'élevage intensif d'ovins, d'où l'importance économique de l'existence d'un foyer actif de fasciolose. Les autres secteurs prospectés n'ont révélé que de rares gîtes à limnées ; par exemple, le réseau dense des affluents du Chélif ne nous a livré qu'une seule localité positive (oued Fodda : El-Asnam).

\section{Différents types de gîtes à Lymnaea truncatula}

D'un point de vue physionomique, nous avons pu classer les gîtes à limnées en quatre types : bords d'oueds permanents à faible courant (19), sources (7), canaux

Fig. I. - Répartition de Lymnaea truncatula dans l'Ouest algérien

I Source M'Sila ; 2 Cap Blanc ; 3 El-Ançor ; 4 Oued Sarno ; 5 Tessala ; 6 Oued Aii ; 7 Source Ouenguel (Rebahia); 8 Oued d'Ain Sultan; 9 Oued Saida (Ain El-Hadjar); ro El-Kreider ; I I Djillali Ben Amar; 12 Rahouia; I3 Sidi Ali Mellal; I4 Misserghin ; I5 Maghnia (source) ; I6 Maghnia (canal); r6 Sebdou (source) ; 18 Sebdou (cascade); I9 Sebdou (canal) ; 20 Ain-Fezza ; 2 I Lamtar ; 22 Bou-Hanifia ; 23 Oued Messaoud (Hennaya) ; 24 Col de Krorchef ; 25 Abdelmalek Ramdane; 26 Oued Bellah; 27 Pont Tafna ; 28 Bel-Abbès'(canal); 29 Tiaret; 30 Col des Zarifètes ; 31 Bel-Hadj ; 32 Oued Sefioum ; 33 Oued Fodda. 


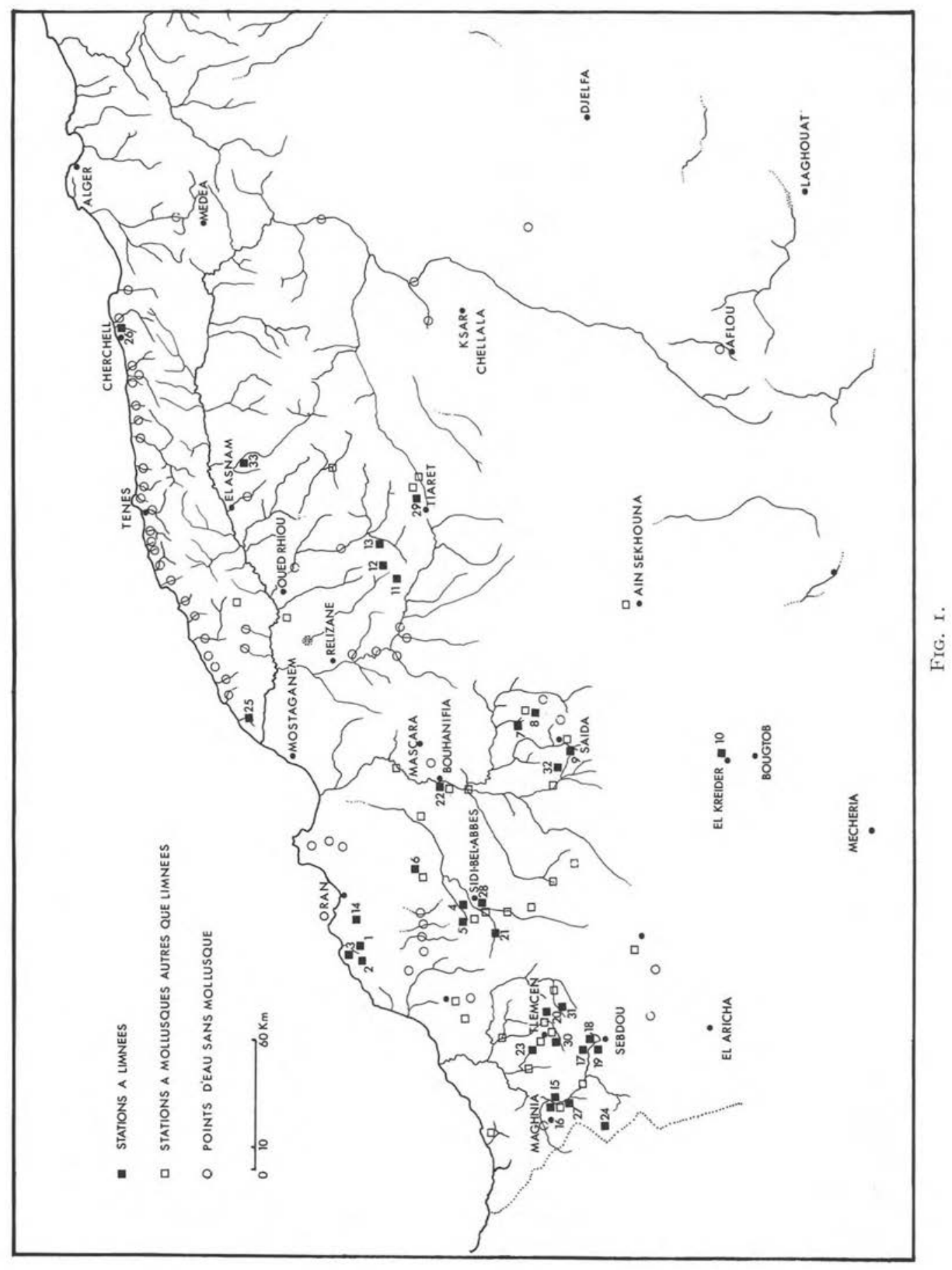


d'irrigation (3), marécages d'extension très variable (4); les zones effectivement occupées par les limnées s'étendent au maximum sur quelques dizaines de $\mathrm{m}^{2}$.

Les oueds typiques présentent au niveau de leur cours principal de fortes variations de débit qui ravinent le lit et ne laissent qu'un fond caillouteux; dans ces milieux, nous n'avons jamais rencontré de limnées, celles-ci ne fréquentant pas les substrats sablonneux et pierreux. Les localités positives se trouvent dans le cours supérieur de certains oueds, là où le courant est faible. Ces Mollusques se trouvent près des bords dans une eau peu profonde $(0$ à $20 \mathrm{~cm})$ et plus ou moins stagnante ; nous avons remarqué la raréfaction des limnées dans les endroits les plus ombragés.

Le ralentissement du courant est une condition de la présence des limnées dans les oueds, que cela soit dû à des prises d'eau (par exemple Misserghin, Bou-Hanifia) ou à la végétation aquatique (Saïda, Oued Sarno, Sidi Ali Mellal).

Les marécages sont généralement alimentés par des sources, éventuellement par les infiltrations d'oueds. Si le substrat est argileux (et dans ce cas souvent piétiné par les ovins ou bovins comme à Bou-Hanifia) ou végétal (c'est-à-dire constitué de débris et de tiges de phragmites), les limnées peuvent être présentes ; par contre les substrats vaseux fluides ne leur sont pas favorables. La distribution des limnées dans les marécages n'est homogène que dans les terrains découverts (Bou-Hanifia); lorsque les arbres existent (El-Kreider), les limnées se trouvent dans les zones éclairées.

Les sources prospectées ont un faible débit : l'eau suinte et s'étale souvent sur une succession de petits plateaux où se forment des dépôts terreux; les limnées vivent sur ces alluvions boueux, évitant le courant principal et les parties sablonneuses.

Les alentours des sources sont généralement boisés, ce qui pose le même problème du manque de luminosité, expliquant probablement la faible densité des populations des sources.

Les canaux d'irrigation, cimentés ou non, dérivent toujours de sources (AinFezza) ou d'oueds (Bel-Abbès, Bel-Hadj, Sebdou). Les limnées trouvent dans certains canaux peu utilisés des conditions favorables à leur développement, montant sur les parois lorsque le niveau de l'eau s'élève. Dans la plupart des cas, les canaux qui nous ont fourni les limnées faisaient suite à des sources ou des oueds eux-mêmes positifs.

\section{Caractères généraux des gîtes à Lymnaea truncatula}

Bien que les localités où nous avons trouvé L. truncatula soient physionomiquement variées, plusieurs caractères communs apparaissent : les stations positives se caractérisent par une eau douce, de faible turbidité, à débit très lent ou lent, par un substrat argileux toujours inondé ou humide, par un éclairement important; les substrats caillouteux ou sablonneux, les vases en décompositions et les zones fortement ombragées sont dépourvues de tout peuplement de Lymnaea truncatula; en ce qui concerne le facteur luminosité, il est très probable qu'il s'explique par son influence sur le développement des algues dont les limnées se nourrissent. Bien que nous 
n'ayons pas effectué une analyse démographique des populations de Limnées, nous avons noté que celles-ci présentent un maximum au printemps (avril, mai) et en automne (octobre, novembre) ; en hiver, les limnées sont rares, en été, elles sont très difficiles à trouver. Dans les canaux, les populations, probablement mieux protégées contre les aléas climatiques, nous ont paru nettement plus stables.

\section{Association de Lymnaea truncatula avec d'autres espèces de Mollusques}

Les physes (Physa acuta Drap.) et les Pseudamnicoles (Pseudamnicola dupotetiana P.) coexistent souvent avec $L$. truncatula mais leur démographie subit des fluctuations différentes ; à Bou-Hanifia par exemple, il y a prédominance de physes et de Pseudamnicoles en automne. Par contre, les limnées ne coexistent pas ou très rarement avec Melanopsis praemorsa L. ; dans plusieurs sources nous avons noté que la partie la plus en amont, envahie par les Melanopsis, était désertée par les limnées, tandis qu'un peu plus en aval, les limnées apparaissent. Cette observation rejoint celle de Dupouy et al. (1980) qui ont noté que les Menalopsis tendent à éliminer progressivement les Basommatophores tel que Planorbarius metidjensis Forbes et Bulinus truncatus Audoin.

\section{Polymorphisme}

Les limnées récoltées dans les différentes localités présentent un polymorphisme de la coquille qui se traduit notamment par la longueur totale, par exemple jusqu'à $12,5 \mathrm{~mm}$ à Tiaret et Saïda, jusqu'à $6 \mathrm{~mm}$ seulement à Bou-Hanifia.

Une étude du polymorphisme enzymatique consacrée à des limnées de neuf de nos stations a fourni des résultats intéressants (Balbo et al., 1982) : pour un enzyme donné les différents allèles possibles peuvent être présents dans la même population avec des proportions variables ; dans d'autres cas, un seul des allèles paraît caractériser toute la population. Nous ne citerons pour exemple que les stations de Bou-Hanifia et de Lamtar, pourtant relativement proches, où l'hydroxy-butyrate deshydrogénase- 2 est représentée à $100 \%$ par deux allèles différents. Ce résultat milite en faveur d'un isolement important des populations que nous avons étudiées. Nous ajouterons que ce travail a montré l'absence d'hétérozygote sur un total de 180 limnées étudiées (20 par station) ce qui démontre une autofécondation régulière sinon exclusive de Lymnaea truncatula en Algérie.

\section{Réceptivité à Fasciola hepatica de quelques souches algériennes de Lymnaea truncatula}

L'hétérogénéité des fréquences des allèles d'enzymes dans les populations de Lymnaea truncatula algériennes nous a amenés à tester leur réceptivité à Fasciola hepatica. Nous avons considéré huit localités : Pont Tafna (Maghnia), Ain-Fezza 
(Tlemcen), Bel-Hadj (Tlemcen), Sebdou, Bel-Abbès, Bou-Hanifia, Ouenguel (Saïda) et Abdelmalek Ramdane (Mostaganem).

La période prépatente étant de 28 jours dans des conditions expérimentales voisines des conditions naturelles, nous avons considéré comme saines les limnées récoltées sur le terrain, gardées au laboratoire pendant un mois et n'ayant émis aucune cercaire au bout de ce laps de temps.

Les œufs de $F$. hepatica ont été obtenus à partir de douves prélevées sur des foies de bovins de l'abattoir d'Oran et maintenues dans du sérum physiologique à $38^{\circ} \mathrm{C}$; les œufs lavés puis incubés à l'obscurité dans de l'eau de source à 24-26 $\mathrm{C}$ éclosent à partir du $17 \mathrm{e}$ jour. Les limnées (taille 3 à $6 \mathrm{~mm}, 15$ à 20 par station) ont été exposées isolément à un miracidium chacune. L'émission des cercaires a été testée au bout d'un mois. Dans le cas des huit stations nous avons obtenu une émission cercarienne pour un nombre de limnées représentant 5 à $20 \%$ des individus exposés. On peut déduire de ce résultat que les $L$. truncatula d'Algérie, bien que formant des populations isolées qui présentent certainement des différences assez importantes du point de vue génétique, sont comparables quant à leur aptitude à héberger les stades larvaires de $F$. hepatica.

\section{Conclusion}

Notre enquête montre que, contrairement aux données jusqu'ici disponibles, le vecteur de la distomatose hepato-biliaire à Fasciola hepatica est très répandu sur le territoire algérien, même si les gites sont toujours de faibles dimensions, nettement isolés les uns des autres et regroupés de façon dominante dans certains bassins hydrographiques. Lymnaea truncatula apparaît urbiquiste dans la mesure où des gîtes physionomiquement très divers peuvent l'accueillir, mais les caractères communs à ces gites prouvent que son écologie est tout de même très comparable dans l'aire de notre enquête. Distribution et cycles saisonniers apparaissent très différents de ce qui est connu pour cette limnée en Europe. Malgré un polymorphisme de la coquille et un polymorphisme enzymatique, la réceptivité de toutes les souches que nous avons testées démontre la possibilité de la transmission de la fasciolose dans des environnements écologiques très divers en Algérie.

\section{BIBLIOGRAPHIE}

Balbo T., Horr K., Lanfranchi P., Massot M., Orecchia P., Paggi L., Nascetti G., BulLINI L. : Studi elettroforetici su popolazioni naturali di Lymnaea (Galba) truncatula. Atti del XI Congresso della Societa Italiana di Parassitologia Camerino, 8-I2 septembre I98I (sous presse).

Bourguignat M. J. R. : Paléontologie des Mollusques terrestres et fluviatiles de 1'Algérie. I862, I $26 \mathrm{p}$.

Dupouy J., AвDelhak F., Yazid F. : Compétition interspécifique entre Melanopsis praemorsa L. (Prosobranchia : Thiaridae) et certains Basommatophores en Oranie et au Sahara NordOccidental. Perspective d'application à la lutte préventive contre la bilharziose. J. Moll. Stud., I980, 46, I-I2. 
Guy Y., Khati B., Rocha E., Le Coroller Y., Ouffriha A., AdAki K. : Distomatose hépatique à Fasciola hepatica. A propos d'un cas. Arch. Inst. Pasteur Algérie, 1969, I, 67-73.

Lik̀vre H. : La répartition de la distomatose algérienne et ses variations. Thése médecine, Alger, 1932, 00, 42-44.

Pallary P. : Faune malacologique du Grand Atlas. J. Conchyl., 1921, 66, 87-1 54 .

Pallary P. : Complément à la faune malacologique de la Berbérie. J. Conchyl., 1926a, 70, I-50.

Pallary P. : Sur une faunule aquatique pliocénique d'Oran. Bull. Soc. Hist. Nat. Afr. Nord,

I926b, $I 7,284-289$.
PALLARY P. : Complément à la faune malacologique de la Berbérie (suite). J. Conchyl., 1927, 71 ,

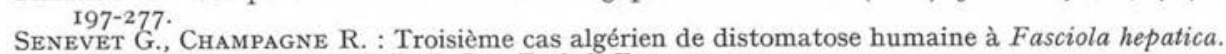
Bons effets du Stovarsol. Bull. Soc. Pathol. Exo., 21, 222-224.

Senevet G., Champagne R. : A propos d'un cas de distomatose à Fasciola hepatica. Arch. Inst. Pasteur Algérie, 1929, 7, 207-216. 